\title{
La antigua displasia broncopulmonar organizada en la era de la nueva displasia broncopulmonar
}

\section{Old organized bronchopulmonary dysplasia in the era of the new bronchopulmonary dysplasia.}

Sancho-Hernández $\mathrm{R}^{1}$, Rojas-Maruri $\mathrm{M}^{2}$, Solorio-Rodríguez $\mathrm{L}^{3}$

\section{CASO CLÍNICO}

Varón de 10 meses de edad sin antecedentes heredofamiliares ni personales patológicos de importancia. Producto gemelar de la primera gesta obtenido de 26.5 semanas de gestación sin esquema de esteroide prenatal, con peso de $800 \mathrm{~g}$ y talla de $35 \mathrm{~cm}$ al nacer; Apgar 7-8 que requirió de intubación endotraqueal para aplicación de surfactante. Durante su estancia en la unidad de cuidados intensivos neonatales tuvo 9 días de ventilación mecánica convencional (VMC) y 33 días de ventilación no invasiva con presión positiva continua de la vía aérea (CPAP) y sepsis neonatal temprana (aislamiento de secreción endotraqueal con Ureaplasma, recibió macrólido por 14 días). Egresó sin aporte de oxígeno suplementario y con reporte de disfunción cerebral generalizada con apneas obstructivas y centrales por estudio polisomnográfico. Con alimentación al seno materno a libre demanda, esquema de inmunización completo, con regurgitación desde los tres meses de vida, vómitos y postura de Sandifer que mejora con el uso inhibidor de bombas de protones y procinético.

Padecimiento y exploración física: acudió a urgencias por estridor bifásico con dificultad respiratoria, respuesta favorable a nebulizaciones con adrenalina, con peso de $4.7 \mathrm{~kg}(\mathrm{p}<5)$, talla $54 \mathrm{~cm}(p<5)$, perímetro cefálico $38 \mathrm{~cm}(\mathrm{p}<5), \mathrm{FC}$ de $130 \mathrm{pm}$,
${ }^{1}$ Servicio de Neumología y Cirugía de Tórax. ${ }^{2}$ Departamento de Patología.

${ }^{3}$ Pediatra Neonatólogo. Pediatría Integral y Terapia Respiratoria.

Instituto Nacional de Pediatría.

Recibido: 14 de abril del 2016

Aceptado: 20 de abril del 2016

Correspondencia

Dr. Rogelio Sancho Hernández Instituto Nacional de Pediatría Insurgentes Sur 3700-C Col. Insurgentes Cuicuilco Cel. 044 (55) 52529997 saherog@yahoo.com.mx

Este artículo debe citarse como Sancho-Hernández R, Rojas-Maruri M, SolorioRodríguez L. La antigua displasia broncopulmonar organizada en la era de la nueva displasia broncopulmonar. Acta Pediatr Mex 2016;37(3):165-174. 
FR de 40 pm y $\mathrm{T}$ de $36.9^{\circ} \mathrm{C}$, tiraje intercostal, retracción supraesternal, espiración prolongada, ruidos cardíacos rítmicos y sin soplos, resto normal. Ante criterios diagnósticos para enfermedad pulmonar obstructiva crónica del lactante por displasia broncopulmonar, enfermedad por reflujo gastroesofágico y posible obstrucción mecánica de la vía aérea superior, se apoyó con esteroide inhalado, procinético y omeprazol, se programó para broncoscopía y endoscopía digestiva superior.

\section{Abordaje multidisciplinario}

Neumología y cirugía de tórax: estudio endoscópico con laringomalacia grado I, estrechez subglótica concéntrica edematosa que obstruía $80 \%$ de la luz de la vía aérea, mucosa granular e irregular que requirieron la ejecución y vigilancia de traqueostomía sin complicaciones, egresó a su domicilio con saturación de $\mathrm{O}_{2}$ de $88 \%$ al aire ambiente que mejoró con aporte de oxígeno a $1 \mathrm{~L} / \mathrm{min}$ a $94 \%$; con estabilidad respiratoria persisten los datos patológicos de reflujo gastroesofágico y en el control endoscópico de seguimiento se advierten: aritenoides edematosas sin colapso de la vía aérea, remisión de la estenosis subglótica sin dificultad para avanzar broncoscopio de $3.6 \mathrm{~mm}$, granuloma en región subglótica y alrededor del estoma que no obstruyen la vía aérea, panendoscopía con datos de esofagitis del tercio superior, se tomaron biopsias y producto de aspirado bronquial para búsqueda de lipófagos y considerar, según resultados, candidato a cirugía antirreflujo por datos de aspiración (Figura 1A).

Infectología: acudió a urgencias por tos, fiebre de $38.5^{\circ} \mathrm{C}$ con datos de síndrome de respuesta inflamatoria sistémica y dificultad respiratoria, saturación de $82 \%$ y radiografía torácica con consolidación basal derecha compatible con neumonía adquirida comunitaria, recibió manejo con penicilina G sódica cristalina, oxígeno suple- mentario y broncodilatador inhalado, evolucionó con insuficiencia respiratoria y deterioro neurológico que requirió apoyo ventilatorio mecánico con cánula endotraqueal y se ocluyó estoma de traqueostomía con parche adhesivo (Figura 1B).

Terapia intensiva: evolucionó con incremento de parámetros ventilatorios con Peep 15, Pip 30, FR 55 pm, $\mathrm{FiO}_{2} 100 \%$, oximetría 93\% e inestabilidad hemodinámica con saturación venosa central de $57 \%$, acidosis respiratoria y síndrome de dificultad respiratoria aguda secundaria a neumonía nosocomial. Se incrementó la cobertura antibiótica a cefepime más vancomicina. Se presentó neumotórax bilateral secundario a barotrauma y se insertaron sondas pleurales bilaterales conectadas a sello de agua y succión negativa, fuga aérea persistente, se agregaron extrasístoles ventriculares con imagen de bloqueo de rama derecha y ecocardiografía con hipertensión pulmonar moderada $(44 \mathrm{mmHg}$ ) secundario al daño pulmonar, se aportó apoyo inotrópico con milrinona, acidosis respiratoria persistente con parámetros ventilatorios para iniciar ventilación de alta frecuencia oscilatoria VAFO (no se pudo contar con el mismo), presentó paro cardiorrespiratorio con respuesta a la reanimación avanzada a los 6 minutos (Figura 2A).

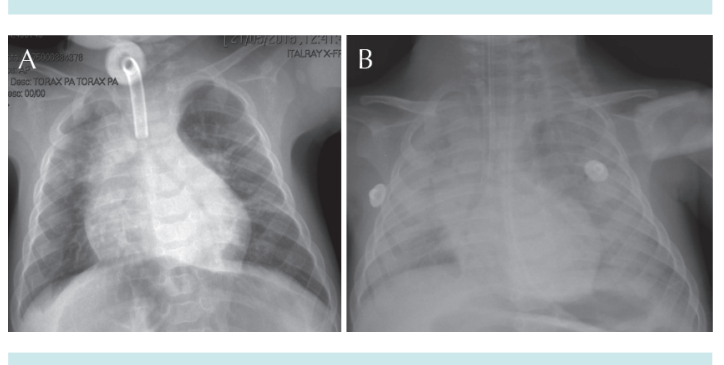

Figura 1. A) Estado post traqueostomía con datos radiológicos de atrapamiento aéreo; patrón bronquítico apical e intersticial difuso característicos de la displasia broncopulmonar. B) Consolidación basal y parahiliar derecha compatible con neumonía comunitaria que evoluciona a sepsis y síndrome de dificultad respiratoria. 
Hematología: presentó en primer y tercer falange de mano izquierda áreas de necrosis distal, se integraron datos de coagulación intravascular diseminada y se inició anticoagulación con enoxaparina, transfusión de crioprecipitados y concentrados plaquetarios. Una vez delimitada el área necrótica se procedería a desbridamiento y cobertura cutánea por servicio de cirugía plástica.

Nefrología: ultrasonografía renal normal, sobrecarga hídrica importante con tasa de filtración glomerular de $38 \mathrm{~mL} / \mathrm{min} / 1.73$ compatible con insuficiencia renal aguda de etiología prerrenal (FeNa 0.2\%) con imposibilidad de iniciar hemodiálisis por inestabilidad hemodinámica.

Laboratorios: hemoglobina de $11 \mathrm{~g} / \mathrm{dL}$, leucocitos $22,800 / \mathrm{mm}^{3}$, plaquetas $23,000 / \mathrm{mm}^{3}$, fibrinógeno 101, dímero D 7.09, tiempo de protrombina de 13 con $74 \%$ de actividad, tiempo parcial de tromboplastina no coagula, creatinina $2.4 \mathrm{mg} / \mathrm{dL}, \mathrm{BUN} 72.7 \mathrm{mg} / \mathrm{dL}, \mathrm{Na}$ 134, K 3.9, Ca 8.2, gasometría arterial con $\mathrm{pH}$ de 6.9, $\mathrm{PO}_{2} 37.5, \mathrm{PCO}_{2}$ 123.9, $\mathrm{HCO}_{3} 28.7$, lactato 0.62, saturación $67 \%$, BT $0.99 \mathrm{mg} / \mathrm{dL}$, BD $0.31 \mathrm{mg} / \mathrm{dL}, \mathrm{BI} 0.68 \mathrm{mg} / \mathrm{dL}$, DHL $2363 \mathrm{mg} /$ dL, AST $208 \mathrm{mg} / \mathrm{dL}$, ALT $88 \mathrm{mg} / \mathrm{dL}$, albúmina $1.6 \mathrm{~g} / \mathrm{L}$.

Presentó un segundo paro cardiorrespiratorio que respondió a reanimación avanzada de 5 minutos, evolucionó con datos de tamponamiento cardíaco y por punción guiada por ecocardiografía se obtuvo aire y sangre escasa, con fracción de eyección ventricular de 15\% se agregó adrenalina y dobutamina a la terapia inotrópica, persistió la fuga aérea con neumotórax y neumomediastino por barotrauma, evolucionó a falla orgánica múltiple con un tercer paro cardiorrespiratorio que no respondió a las maniobras de reanimación avanzada (Figuras 2B y 2C).

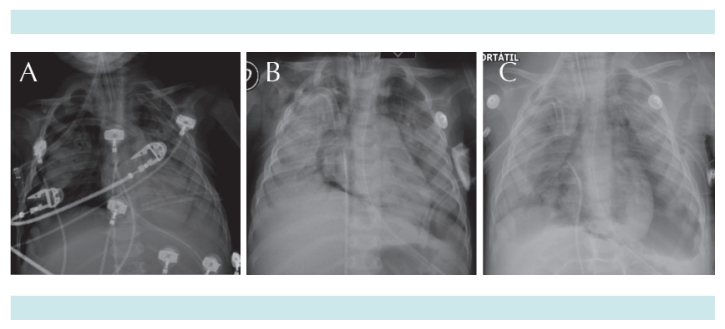

Figura 2. A) Neumotórax bilateral y recurrente secundario al volu-barotrauma y sepsis pulmonar subyacente. B) Persistencia de fuga aérea con neumomediastino y tamponamiento cardíaco clínico e hiperinsuflación pulmón izquierdo. C) Sobredistensión pulmonar bilateral sin datos de fuga aérea con enfisema intersticial persistente previo al fallecimiento por SDRA y sepsis

\section{COMENTARIO ANATOMOPATOLÓGICO}

\section{Dr. Mauricio Rojas Maruri}

En la autopsia realizada se observó externamente estoma de traqueotomía, mano izquierda con coloración violácea del $1^{\circ}, 2^{\circ}, 3^{\circ}$ y $4^{\circ}$ dedos. A la visualización de órganos, la laringe presentaba mucosa amarilla de aspecto granular y opaca, la tráquea se observaba congestiva con tapón de moco de color amarillo mezclado con sangre fresca que ocluía su luz con disminución en su calibre (Figura 3A).

Al corte los pulmones se observaron de aspecto congestivo, así como área de aspecto hemorrágico localizada en lóbulo superior de pulmón derecho (Figura 3B). El corazón se observaba ligeramente dilatado a expensas de cavidades derechas.

Los cortes histológicos de la tráquea mostraron epitelio ulcerado con depósitos de fibrina, tejido de granulación, inflamación aguda y crónica, con área extensa de metaplasia escamosa (Figura 4).

El pulmón mostró abundante moco en sus luces bronquiales y bronquiolares, los alveolos presentaban abundantes macrófagos en su luz 


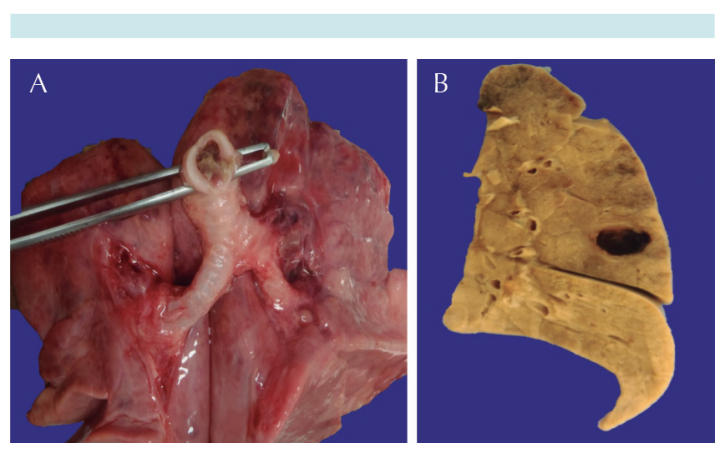

Figura 3. A) Aspecto congestivo de los pulmones y tapón de moco que ocluía la luz de la tráquea. B) Vista posterior de corte de pulmón derecho que muestra área nodular de aspecto hemorrágico en el lóbulo superior.

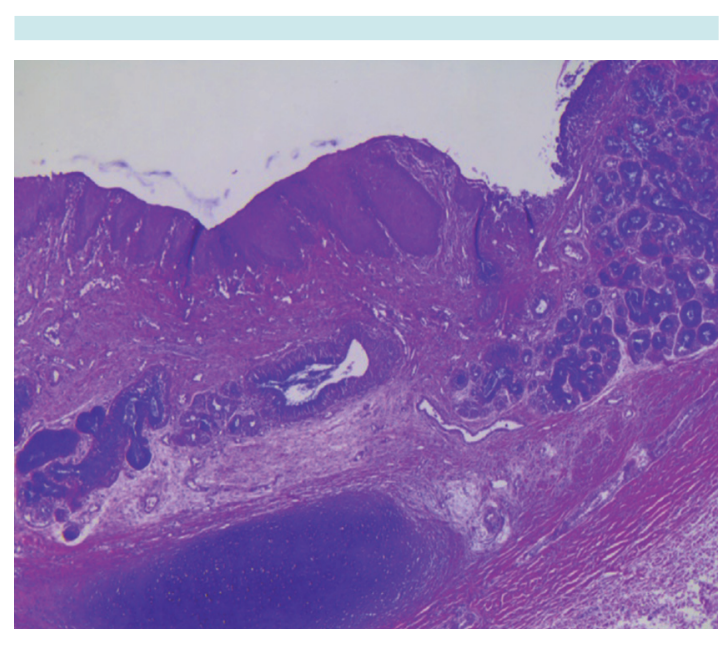

Figura 4. Transformación a epitelio plano estratificado del epitelio respiratorio.

entremezclados con polimorfonucleares, linfocitos y material eosinófilo denso (Figura 5A), así como engrosamiento moderado de la pared (Figura 5B).

El intersticio mostró fibrosis moderada a la tinción de Masson e infiltrado inflamatorio leve, compuesto por linfocitos (Figura 6A). Los vasos presentaron ligero engrosamiento de la pared

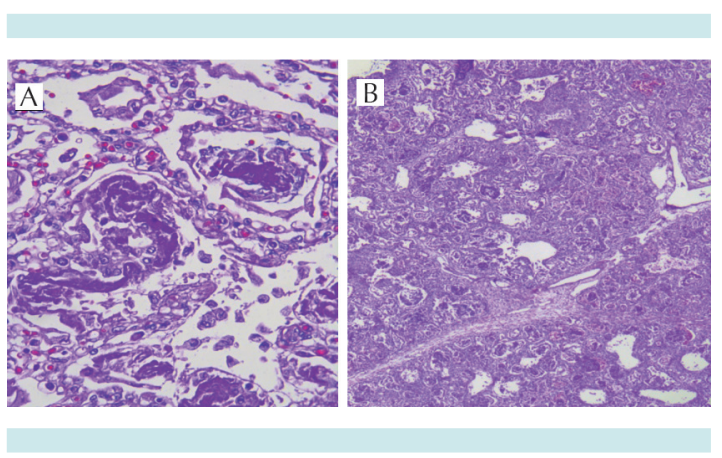

Figura 5. A) Alveolos con material eosinófilo denso en su luz. B) Pérdida de luces bronquiales y bronquiolares.

y se identificó vaso arterial de mediano calibre con trombo intraluminal (Figura 6B).

Otros hallazgos encontrados fueron la presencia de úlceras esofágicas, inflamación aguda y crónica en epitelio de la vejiga, vacuolización de túbulos renales, hipereosinofilia de músculo estriado y cambios cerebrales caracterizados por cambios espongióticos, picnosis de células gliales y neuronales, así como áreas de hemorragia.

Los hallazgos en el pulmón de esta autopsia actualmente son difíciles de observar; esto debido principalmente a la implementación de nuevas terapéuticas y porque generalmente los niños con esta patología, de ser grave, en ocasiones no progresan a la cronicidad debido a una muerte prematura. Es por esto que en los

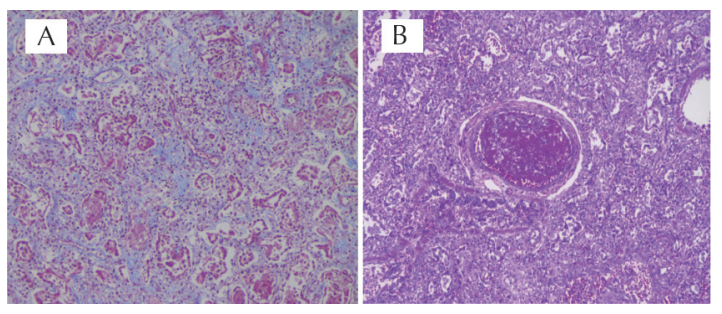

Figura 6. A) Tinción de Masson que muestra la fibrosis. B) Trombo en vaso de mediano calibre. 
foros de expertos se llegó a hablar de una displasia broncopulmonar "vieja" y una "nueva". El hallazgo de hemorragia pulmonar focal es una complicación de la punción torácica realizada por la presencia de neumotórax. La necrosis de las falanges de la mano izquierda y los trombos pulmonares nos hablan de una complicación trombótica de la arterioclisis.

\section{Diagnósticos anatómicos principales}

Displasia broncopulmonar en fase de organización. Laringitis y traqueítis aguda y crónica leve ulcerada con metaplasia escamosa extensa con hemorragia antigua, hiperplasia de glándulas mucosas y tejido de granulación (historia clínica de estenosis subglótica del $80 \%$ y colocación de traqueostomía). Datos de sobrecarga de cavidades derechas e hipertensión pulmonar; datos histológicos de broncoaspiración; tromboembolia pulmonar y hemorragia pulmonar focal en lóbulo superior derecho (historia clínica de neumotórax con punción torácica). Encefalopatía hipóxico isquémica con hemorragia parenquimatosa focal del lóbulo cerebral parietal derecho; necrosis de falanges distales de $1^{\circ}, 2^{\circ}$, $3^{\circ}$ y $4^{\circ}$ dedo de mano izquierda (arterioclisis); úlceras esofágicas; cistitis hemorrágica; peso bajo para la edad; datos anatómicos de choque (necrosis tubular aguda y miopatía hipóxico isquémica).

\section{DISCUSIÓN}

\section{Dr. Rogelio Sancho Hernández}

La displasia broncopulmonar es una enfermedad pulmonar crónica que ocurre principalmente en recién nacidos pretérmino con peso menor de 1,000 g y de <28 semanas de gestación. Estos pacientes clásicamente son tratados con presiones ventilatorias y concentraciones de oxígeno altas, en el contexto de un síndrome de dificultad respiratoria. ${ }^{1}$ El debut clínico, radiológico y patológico determinaron hacia 1967 con Northway la afección descrita como la "antigua" displasia broncopulmonar caracterizada, como en nuestro caso, por bronquiolitis necrotizante, vasculopatías con hipertensión pulmonar, infiltrado inflamatorio, áreas alternantes de sobredistensión y atelectasias con marcada fibrosis, lesión epitelial e hiperplasia muscular de la vía aérea y el parénquima pulmonar $^{2,3}$ (Figura 7A). Aunque la mortalidad asociada a estos prematuros se ha alterado significativamente por el uso de corticosteroides prenatales, surfactante postnatal, innovación en las estrategias ventilatorias y nutricionales, el riesgo de desarrollar morbilidad respiratoria crónica a largo plazo permanece aún muy alto en estos sobrevivientes. Estos avances en la neonatología no sólo han modificado desde 1979 con Bancalari los criterios de diagnóstico y de morbilidad respiratoria tras el alta de las unidades neonatales, sino que han definido una etapa de la "nueva" displasia broncopulmonar ${ }^{1,3-4}$ (Figura 7B). Aunque las presentaciones severas de la antigua forma de displasia broncopulmonar están siendo reemplazadas por las presentaciones leves de las nuevas formas, los expertos están convencidos que el desarrollo de la displasia broncopulmonar conlleva un proceso patogénico multifactorial de inmadurez del tejido pulmonar, barotrauma y volutrauma por ventilación mecánica, lesión oxidativa y mediadores pro- y antiinflamatorios, donde dichos procesos representan las dianas para dirigir las estrategias terapéuticas comunes a ambos tipos de displasia broncopulmonar. ${ }^{5}$

¿Qué insultos patogénicos de la etapa neonatal pueden ser advertidos en nuestro caso clínico de discusión?

a) Sepsis bacteriana asociada a un 35 a $62 \%$ con displasia broncopulmonar es también un fuerte indicador de enfermedad pulmonar crónica. ${ }^{6}$ 


A
\begin{tabular}{|c|c|}
\hline $\begin{array}{c}\text { Antigua DBP } \\
\text { sobredistensión y } \\
\text { atelectasias }\end{array}$ & $\begin{array}{c}\text { Hipoplasia alveolar, } \\
\text { disminución de la } \\
\text { complejidad acinar }\end{array}$ \\
\hline $\begin{array}{c}\text { Lesión epitelial de la vía } \\
\text { aérea severa }\end{array}$ & $\begin{array}{c}\text { Hiperplasia de músculo } \\
\text { liso variable }\end{array}$ \\
\hline $\begin{array}{c}\text { Hiperplasia de } \\
\text { músculo liso }\end{array}$ & $\begin{array}{c}\text { Hiperplasia de músculo } \\
\text { liso variable }\end{array}$ \\
\hline $\begin{array}{c}\text { Lesión hipertensiva } \\
\text { vascular pulmonar }\end{array}$ & $\begin{array}{c}\text { Menor vasculopatía } \\
\text { arteriolar pulmonar }\end{array}$ \\
\hline $\begin{array}{c}\text { Disminución de la } \\
\text { superficie interna aérea y } \\
\text { alveolar }\end{array}$ & $\begin{array}{c}\text { Disminución de capilares } \\
\text { dismórficos }\end{array}$ \\
\hline
\end{tabular}

B

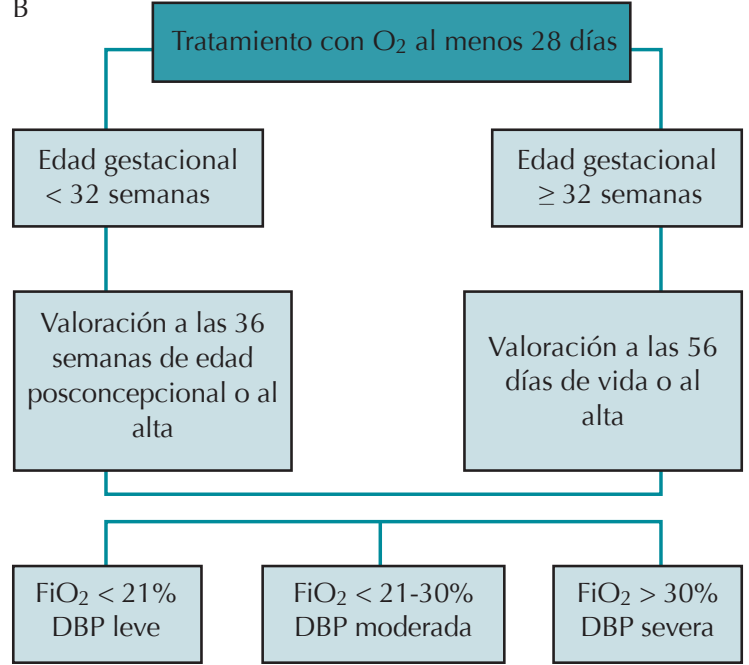

Figura 7. A) Datos histopatológicos que distinguen la antigua de la nueva displasia broncopulmonar. B) Actual definición y clasificación operativa de la displasia broncopulmonar. DBP: displasia broncopulmonar.

b) Colonización con Ureaplasma, que correlaciona la corioamnioitis materna con su presencia en membranas, mucosas y tracto respiratorio de los pacientes afectados con displasia broncopulmonar. ${ }^{7}$

c) Ventilación mecánica y toxicidad por oxígeno con el consecuente trauma endobronquial, sobredistensión de la vía aérea, detención del desarrollo pulmonar y detonadores de la cascada inflamatoria.

d) La presencia de un conducto arterioso persistente con repercusión hemodinámica también ha mostrado asociación con la displasia broncopulmonar. ${ }^{8}$

Todo lo anterior representan los actuales objetos de acción preventiva y terapéutica, a saber: corticosteroides prenatales como inductores de madurez pulmonar y para disminuir el riesgo de displasia broncopulmonar y de extubación temprana en las primeras 2 semanas de vida; azitromicina en su acción antibiótica y antiinflamatoria contra agentes atípicos; vitamina A, E y selenio profilácticos para inducción de procesos de reparación y daño inducido por oxidación; cafeína, pentoxifilina y acetilcisteína con impacto controversial como métodos de prevención de displasia broncopulmonar; y desde luego las estrategias ventilatorias y la terapia surfactante en sus modalidades de intubación-surfactanteextubación con presión positiva continua de la vía aérea nasal (INSURE), hipercapnia permisiva y ventilación gentil; la restricción hídrica y la fortificación calórica en el aspecto nutricional, entre otros. ${ }^{3}$

¿Qué elementos de morbilidad impactaron en el desenlace post neonatal de nuestro paciente?

Al egreso de la unidad neonatal el manejo multidisciplinario es mandatorio si consideramos a la displasia broncopulmonar como una enfermedad crónica multisistémica, con altos índices de rehospitalización durante el primer año, persistencia de patología obstructiva y reactiva de la vía aérea, enfisema secuelar intersticial y diversos grados de afección en el crecimiento, 
sistema cardiovascular, neurodesarrollo y energético; ${ }^{9,10}$ tal como pudimos corroborarlo en las comorbilidades acompañantes de nuestro paciente tras el egreso de la unidad neonatal: enfermedad por reflujo gastroesofágico con potenciales criterios de cirugía antirreflujo, desnutrición e inmunosupresión secundarias, obstrucción mecánica (estenosis subglótica) y dinámica (malasia y discinesia traqueobronquial) de la vía aérea, hipertensión pulmonar y lesión endotelial y sistémica asociada a la hipoxemia y daño pulmonar crónico como causas del desenlace fatal.

Establecido el diagnóstico de displasia broncopulmonar el pediatra se encuentra con una serie de conflictos y controversias en torno a la terapéutica y evidencia científica existente: esteroides inhalados con beneficio local, pero con impacto negativo en el neurodesarrollo; ${ }^{11}$ diuréticos con impacto favorable en el manejo del edema alveolar e intersticial, pero con repercusiones metabólicas e hidroelectrolíticas importantes; ${ }^{12}$ terapia broncodilatadora inhalada que si bien mejoran la función pulmonar y son pilares en el manejo de las exacerbaciones agudas de la displasia broncopulmonar, podrían empeorar las obstrucciones dinámicas de la vía aérea. ${ }^{13,14}$ Parece ser aceptable la oxigenoterapia domiciliaria para disminuir la hipertensión pulmonar y la sobrecarga ventricular derecha manteniendo saturaciones $\geq 94 \%$, es razonable aceptar que la selección y la toma de decisiones debe ser entonces individualizada.

El sistema broncopulmonar como órgano de choque en la displasia broncopulmonar se traduce en un riesgo mayor de sibilancias recurrentes, así como una susceptibilidad de $80 \%$ a infecciones respiratorias víricas de vías aéreas inferiores (el lactante pudo ser candidato a la profilaxis con palivizumab frente al virus sincitial respiratorio); el evento neumónico comunitario fue el motivo de último ingreso y desencadenante inicial de las complicaciones sépticas y por volu-barotrauma asociada a las presiones inspiratorias elevadas de la ventilación mecánica convencional. ${ }^{15} \mathrm{La}$ confluencia de factores infecciosos y ventilatorios en el pulmón inmaduro con displasia broncopulmonar favorecen a entidades que condicionan un atrapamiento ectópico de aire en el intersticio pulmonar de forma difusa, localizada o más frecuentemente mixta y al que se ha denominado enfisema secuelar intersticial; éste concepto permite incluir y orientar las diversas alteraciones radiológicas denominadas como hiperinsuflación, enfisema buloso, enfisema intersticial persistente y barotrauma que afectan al pulmón y cuya traducción clínica y funcional resulta imprecisa originando términos múltiples, que conllevan a confusión diagnóstica y terapéutica. Por lo anterior, se propone una clasificación con un enfoque clínico-radiológico-funcional que permita una orientación diagnóstica en tres grupos y determinar la mejor opción de tratamiento conservador o quirúrgico en base al pronóstico funcional, la morbilidad y mortalidad en los resultados. Así tenemos:

Grupo I. Corresponde a la variante de hiperinsuflación obstructiva y la denominamos enfisema lobar intersticial adquirido.

Grupo II. En el que se ubican los recién nacidos con antecedentes de ventilación mecánica convencional, así como sus complicaciones asociadas y cuyas alteraciones quísticas pulmonares persistieron por más de 4 semanas con evolución subaguda, la denominamos enfisema intersticial neonatal.

Grupo III. Donde se ubican la población con antecedentes de ventilación mecánica convencional, así como sus complicaciones asociadas: neumotórax, neumo-mediastino, neumoperitoneo y enfisema subcutáneo, cuyas alteraciones persistieron dentro de las primeras 4 semanas del insulto ventilatorio con evolu- 
ción aguda, la Ilamamos enfisema intersticial por barotrauma. ${ }^{16}$

El grupo enfisema lobar intersticial adquirido puede cursar con obstrucción bronquial intraluminal causada por las complicaciones secuelares de la intubación (granulomas, estenosis), o bien representar obstrucciones dinámicas con colapso de la vía aérea (traqueoendobronquitis, discinesia-malacia), procesos que deben ser diagnosticados y tratados con broncoscopía en todos los casos; el tratamiento quirúrgico debe considerarse en paciente que curse con:

a) Enfermedad localizada en uno o dos lóbulos pulmonares sin respuesta al tratamiento médico y endoscópico.

b) Deterioro progresivo de la función pulmonar a pesar del apoyo ventilatorio.

c) Sobredistensión de un lóbulo pulmonar involucrado con desviación mediastinal significativa.

d) Compresión de tejido pulmonar adyacente con atelectasia, bronquiectasia y destrucción pulmonar. ${ }^{17}$

El grupo de niños con enfisema intersticial neonatal es un fenómeno típicamente transitorio, pero puede persistir como una conglomeración radiológica quística que puede causar dificultad respiratoria progresiva; por guardar un estrecho diagnóstico diferencial con las lesiones congénitas quísticas broncopulmonares, la resección quirúrgica es el tratamiento tradicional; no obstante, deben agotarse en una fase temprana las estrategias conservadoras con ventilación de alta frecuencia (oscilatorias VAFO o jet VAFJ), así como el estricto control sistémico, antiinflamatorio local, farmacológico y nutricional de la displasia broncopulmonar acompañante. La indicación quirúrgica para éstos pacientes se da cuando existe: a) Dificultad respiratoria por desviación mediastinal.

b) Progresión de los síntomas con persistencia o incremento del tamaño de las lesiones.

c) Dificultad para excluir el diagnóstico diferencial con las lesiones quísticas congénitas pulmonares.

d) Exclusión local y funcional por gammagrafía pulmonar de un lóbulo pulmonar generador de cortocircuito vascular pulmonar.

El grupo de enfisema intersticial por barotrauma incluye a sobrevivientes con displasia broncopulmonar sometidos a presiones inspiratorias excesivamente elevadas, en una serie de casos las presiones inspiratorias utilizadas fueron de 29 a $40 \mathrm{~cm} \mathrm{H_{2 }} \mathrm{O}$ con una Peep de 8 a $18 \mathrm{~cm} \mathrm{H}_{2} \mathrm{O}$, y en todos una $\mathrm{FiO}_{2}$ de $100 \%$. El tratamiento del barotrauma depende de la repercusión clínica: para el neumotórax con neumomediastino y neumoperitoneo el tratamiento suele ser conservador con estrategias ventilatorias encaminadas a disminuir las presiones inspiratorias elevadas y favorecer el reclutamiento alveolar, como son las modalidades de alta frecuencia en sus versiones jet u oscilatorias, además de un drenaje pleural con sonda conectada a un sistema de sello de agua y succión negativa; si éste debuta como hipertensión intraabdominal con síndrome compartimental con gran repercusión hemodinámica, es preciso realizar una descompresión abdominal inmediata con paracentesis y colocación de drenaje intraperitoneal; este grupo representa, como en nuestro caso, el grupo con mayor mortalidad en asociación con neumonía-choque séptico y síndrome de dificultad respiratoria aguda ${ }^{16}$ (Figura 8).

El interés por la presente sesión es que los hallazgos histopatológicos de nuestro caso, propios de la forma antigua de displasia broncopulmonar, son infrecuentes de advertir en la nueva era de 


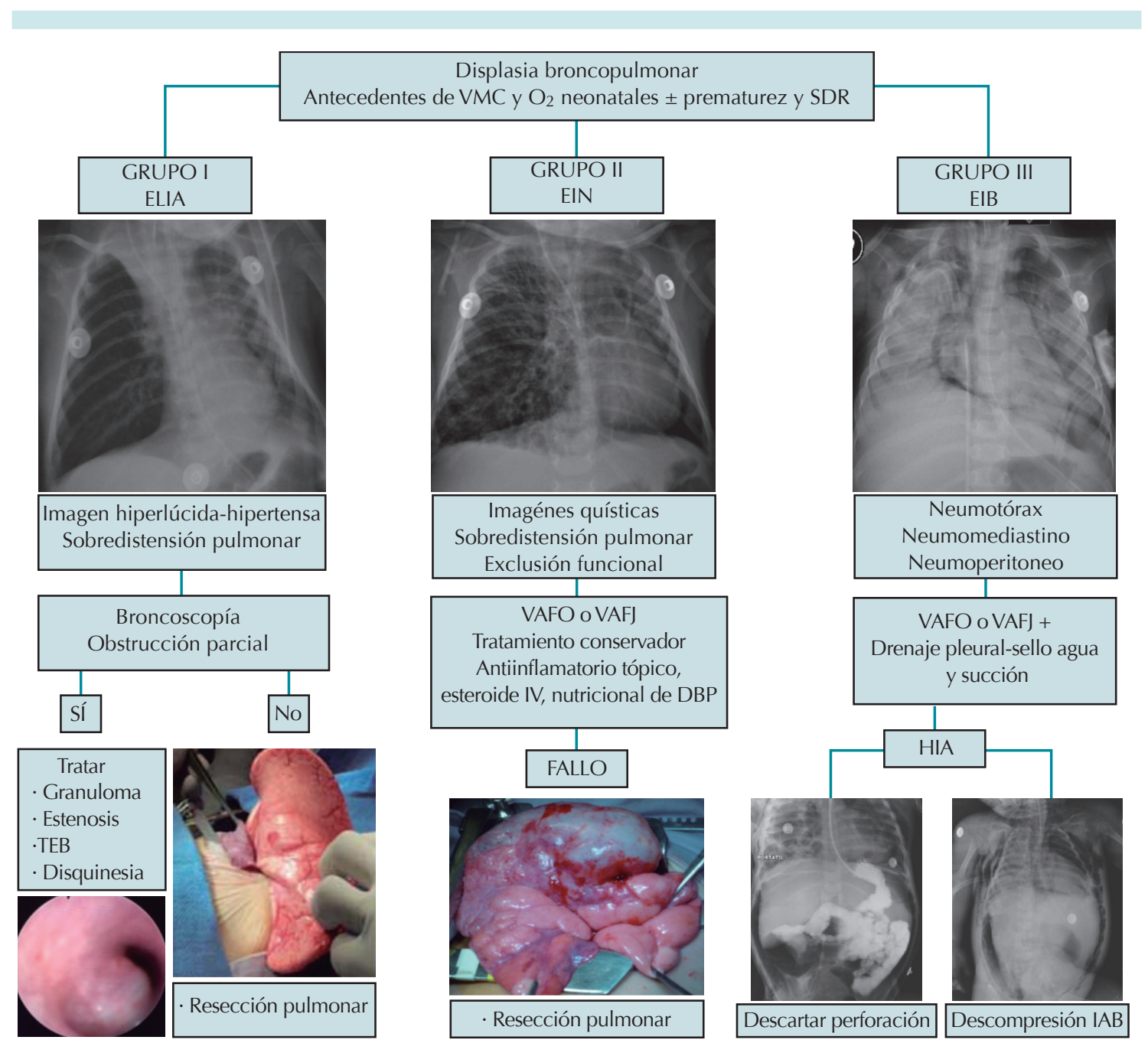

Figura 8. Abordaje de las secuelas enfisematosas intersticiales en pacientes con displasia broncopulmonar. ELIA (enfisema lobar intersticial adquirido); TEB (traqueoendobronquitis); EIN (enfisema intersticial neonatal); VAFO o VAFJ (ventilación alta frecuencia oscilatoria o jet); EIB (enfisema intersticial por barotrauma); HIA (hipertensión intraabdominal); VMC (ventilación mecánica convencional).

las terapéuticas neonatales y más aún exhibir todos los daños de cronicidad pulmonar que sólo eran reconocidos en los productos de autopsia por muertes prematuras asociadas a la displasia broncopulmonar. Lo anterior permite concluir que el desarrollo de la displasia broncopulmonar es un proceso multifactorial resultante de un desequilibrio entre los mecanismos pro- $y$ antiin- flamatorios, que a la confrontación de los nuevos hallazgos a favor de los mecanismos proinflamatorios, las estrategias terapéuticas están dirigidas a promover el control de los mecanismos en el desarrollo alveolar, como los recientes reportes relacionados con la aplicación intratraqueal conjunta de esteroides y surfactantes como medidas preventivas de la displasia broncopulmonar. ${ }^{18-20}$ 


\section{REFERENCIAS}

1. Coalson J. Pathology of new bronchopulmonary displasia. Seminars in Neonatology. 2003;(8):73-81.

2. Northway WH Jr, Rosan RC, Porter DY. Pulmonary disease following respiratory therapy of hyaline membrane disease. Bronchopulmonary dysplasia. N Engl J Med. 1967;276(7):357-68.

3. Kair LR, et al. Bronchopulmonary dysplasia. Pediatrics in Review. 2012;33(6):255-263.

4. Jobe AH, Bancalari E. Bronchopulmonary Dysplasia. Am J Respir Crit Care Med. 2001;163(7):1723-29.

5. Mosca F, Colnaghi M, Fumagalli M. BDP: old and new problems. J Matern Fetal Neonatal Med. 2011;24(Supl 1):80-2.

6. Lahra MM, Beeby PJ, Jeffery HE. Intrauterine inflammation, neonatal sepsis, and chronic lung disease: a 13 year hospital cohort study. Pediatrics. 2009;123 (5):1314-19.

7. Schelonka RL, Katz B, Waites KB, Benjamin DK Jr. Critical appraisal of the role of Ureaplasma in the development of bronchopulmonay dysplasia. Pediatr Pulmonol. 2001; doi 10.1002/ppul.21508

8. Watterberg KL, Scott SM, Backstrom C, et al. Links netween early adrenal function and respiratory outcome in preterm infants: airway inflammation and patent ductus arteriosus. Pediatrics. 2000;105 (2):320-24.

9. Bhandari A, Bhandari V. Piffalls, problems, and progress in bronchopulmonary displasia. Pediatrics. 2009;13(6):15621573.

10. Atkinson SA. Special nutrition needs of infants for prevention of and recovery from bronchopulmonary displasia. J Nutr. 2001;131(3):942 S-946S.

11. Shah V, Ohlsson A, Halliday HL, Dunn MS. Early administration of inhaled corticosteroids for preventing chronic lung disease in ventilated very low birth weight preterm neonates. Cochrane Database Syst Rev. 2007;(4):CD001969.

12. Brion LP, Primhak RA. Intravenous or entereal loop diuretics for preterm infants with (or developing) chronic lung disease. Cochrane Database Syst Rev. 2002;(1):CD001453.

13. Fok TF. Adjunctive pharmacotherapy in neonates with respiratory failure. Semin Fetal Neonatal Med. 2009;14(1):4955.

14. Denjean A, Paris-Llado J, Zupan V, et al. Inhaled salbutamol and beclomethasone for preventing bronchopulmnarydisplasia: a randomiseddouble blind study. Eur J Pediatr. 1998;157(11):926-31.

15. Cruz RJ, Pérez Fernández LF, Herrera GW, Granados NF. Neumopeitoneo causado por ventilación mecánica asistida. Acta PediatrMex. 2010;31(4):168-173.

16. Sancho HR, et al. Enfermedad secuelar enfisematosa intersticial en los niños: propuesta de una clasificación y experiencia en el tratamiento conservador y quirúrgico. Trabajo libre. 75 Congreso Neumología y Cirugía de Tórax. Guadalajara 2016.

17. Sancho HR, Pérez Fernández LF, Cuevas Schacht FJ. Enfisema lobar adquirido durante una neumopatía crónica intersticial: implicaciones quirúrgicas y revisión de la literatura. Acta Pediatr Mex. 2013;34:189-195.

18. Bourbon JR, Boucherat O, Boczkowski, et al. Bronchopulmonary dysplasia and emphysema: in search of common therapeutic targets. Trends in Molecular Medicine. 2009;15(4):169-179.

19. Yeh TF, Chen $\mathrm{CM}, \mathrm{Wu} \mathrm{SY}$, et al. Intratracheal administration of budesonide/surfactant to prevent bronchopulmonary dysplasia. Amer J of Respiratory and Crit Car Med. 2016;193(1):86-95.

20. Bancalari E, Jain D. Prevention of bronchopulmonary dysplasia: are intratracheal steroids with surfactant a magic bullet? 2016;193(1):12. 